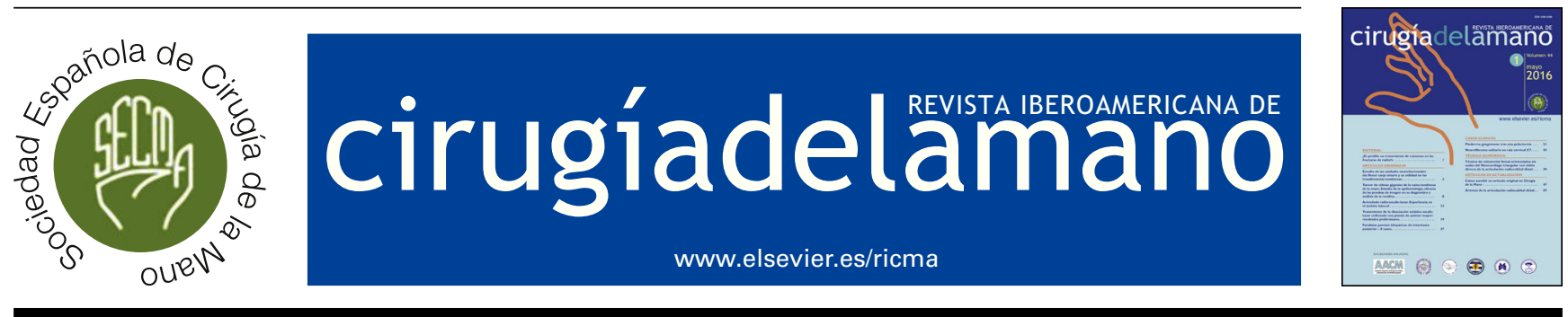

\title{
Osificación heterotópica en codo: resultados tras tratamiento quirúrgico
}

\section{Chaverri Fierro ${ }^{a, *}$ y C. Abellán Miralles ${ }^{b}$}

\author{
a Hospital Asepeyo Sant Cugat, Sant Cugat del Vallès, Barcelona, España \\ b Unidad Codo, Hospital Asepeyo Sant Cugat, Sant Cugat del Vallès, Barcelona, España
}

Recibido el 3 de febrero de 2016; aceptado el 4 de mayo de 2016

Disponible en Internet el 12 de octubre de 2016

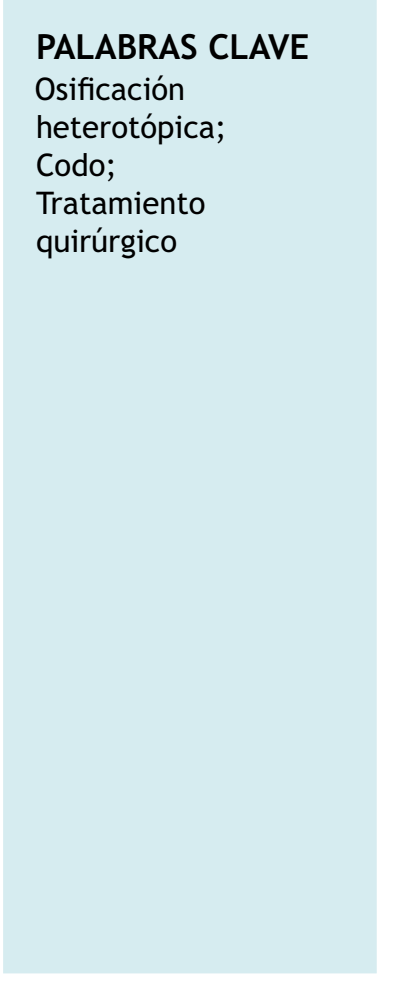

\begin{abstract}
Resumen
Introducción y objetivos: Se entiende osificación heterotópica $(\mathrm{OH})$ como la formación ectópica de hueso maduro en los tejidos blandos adyacentes a zonas previamente lesionadas. La $\mathrm{OH}$ es una de las causas de rigidez articular del codo postraumática, con una prevalencia que oscila según series entre el 3 y el $45 \%$. En su patogénesis se postula la diferenciación de células mesenquimales pluripotenciales en osteoblastos, siendo estos los causantes de la formación de hueso ectópico; sin embargo, se desconocen las causas que provocan esta reacción. Recientemente, diversos estudios de series de casos retrospectivos mediante análisis uni y multivariante han intentado encontrar factores de riesgo (FR) y parámetros predictores de la formación de $\mathrm{OH}$, así como de su repercusión clínica.

Material y métodos: Presentamos un análisis retrospectivo de los casos de $\mathrm{OH}$ en codo tratados quirúrgicamente en nuestro centro entre los años 2005 y 2015. Se recogieron variables demográficas, antecedentes personales, evento traumático inicial, clínica, tiempo desde el traumatismo hasta el diagnóstico de $\mathrm{la} \mathrm{OH}$, tiempo desde el diagnóstico de $\mathrm{OH}$ hasta la cirugía escisional, seguimiento, recurrencia, y grados de movilidad en precirugía escisional y al final del seguimiento. Con ello pretendemos mostrar nuestra experiencia en el tratamiento quirúrgico de esta patología.

Resultados: Obtuvimos 4 casos con un tiempo medio de seguimiento de 40 meses. En 2 casos el evento traumático fue la luxación de codo, y en los otros 2 , la ruptura del tendón del bíceps en su inserción distal. Tres de ellos debutaron clínicamente con limitación en la movilidad articular, y uno con déficit motor a nivel radial. Todos los casos fueron varones con una edad media de 48 años. El tiempo medio transcurrido desde el evento traumático hasta el diagnóstico de $\mathrm{OH}$ fue de 4 meses, realizándose el tratamiento quirúrgico escisional tras el diagnóstico igualmente a los 4 meses aproximadamente como media. Tres de los 4 casos recidivaron, apareciendo nuevas calcificaciones con repercusión clínica y funcional.
\end{abstract}

\footnotetext{
* Autor para correspondencia.

Correo electrónico: danichaverrifierro@gmail.com (D. Chaverri Fierro).
} 
Conclusión: Nuestros resultados convergen con los de la literatura en cuanto a FR y resultados funcionales; sin embargo, llama la atención el alto índice de recidiva en nuestra serie, con una tasa del $75 \%$ frente al $10-24 \%$ descrito en la literatura.

(c) 2016 SECMA. Publicado por Elsevier España, S.L.U. Este es un artículo Open Access bajo la licencia CC BY-NC-ND (http://creativecommons.org/licenses/by-nc-nd/4.0/).

Heterotopic ossification: Outcomes after surgical treatment

\begin{abstract}
Introduction and aims: Heterotopic ossification $(\mathrm{HO})$ is understood as mature ectopic bone formation adjacent to previously injured soft tissues. HO is one of the causes of elbow post-traumatic stiffness with a prevalence ranging between $3 \%$ to $45 \%$. In its pathogenesis differentiation of mesenchymal stem cells into osteoblasts has been postulated, however aetiology remains still unknown. Recently retrospective case series studies using uni and multivariate analysis have been developed in order to find risk factors and predictive parameters of $\mathrm{HO}$ formation as well as its clinical impact.

Material and methods: We present a retrospective analysis of cases of $\mathrm{HO}$ in elbow treated surgically in our center from 2005 to 2015 . Medical history, traumatic event, sign and symptoms, time from trauma to the diagnosis of $\mathrm{HO}$, time from $\mathrm{HO}$ diagnosis to excision surgery, follow up, recurrence, and degrees of mobility pre excisional surgery and at the end of follow up were collected. Our goal is to show our experience in the surgical treatment of this pathology.

Results: 4 cases were obtained with an average follow-up time of 40 months. In two cases traumatic event was elbow dislocation and the other two distal biceps tendon rupture. Three of these present limitation in joint mobility and the other one radial nerve palsy. All cases were men with a mean age of 48 years. The average time since the traumatic event to the diagnosis of HO was 4 months, performing the excisional surgery in the first 4 months after diagnosis equally. Three cases presented recurrence with clinical and functional impact.

Conclusion: Our results are similar with those of the literature related to risk factors and functional outcomes, however we highlight the high recurrence rate in our series, with a rate of $75 \%$ compared to $10 \%-24 \%$ reported in the literature.

(C) 2016 SECMA. Published by Elsevier España, S.L.U. This is an open access article under the CC BY-NC-ND license (http://creativecommons.org/licenses/by-nc-nd/4.0/).
\end{abstract}

\section{Introducción}

Se entiende osificación heterotópica $(\mathrm{OH})$ como la formación ectópica de hueso maduro en los tejidos blandos adyacentes a zonas previamente lesionadas ${ }^{1}$. $\mathrm{La} \mathrm{OH}$ es una de las causas de rigidez articular del codo postraumática, con una prevalencia que oscila según series entre el 3 y el $45 \%$. Este amplio rango de cifras es debido a la variabilidad en la definición de $\mathrm{OH}$, incluyendo algunos estudios pequeñas cantidades de $\mathrm{OH}$ en ligamentos y músculos clínicamente irrelevantes ${ }^{2,3}$. Estudios que incluyeron la relevancia clínica en la definición de $\mathrm{OH}$, mediante la medición de la disminución del rango de movilidad, determinaron una prevalencia entre el 7-20\% de los pacientes tratados quirúrgicamente después de un evento traumático en el codo ${ }^{4,5}$. Morrey y Harter diferenciaron entre $\mathrm{OH}$, miositis osificante y calcificaciones periarticulares, consistiendo las 2 primeras en la formación de hueso ectópico maduro (en el caso de la miositis de localización muscular), y la última en depósitos de pirofosfato cálcico periarticular de morfología varia sin formar una estructura trabecular. Es importante diferenciar estas entidades para no sobrediagnosticar el fenómeno de la $\mathrm{OH}^{6}$.

En su patogénesis se postula la diferenciación de células mesenquimales pluripotenciales en osteoblastos, siendo estos los causantes de la formación de hueso ectópico; sin embargo, se desconocen las causas que provocan esta reacción. Recientemente, diversos estudios de series de casos retrospectivos mediante análisis uni y multivariante han intentado encontrar factores de riesgo y parámetros predictores de la formación de $\mathrm{OH}$, así como de su repercusión clínica. En la tabla 1 mostramos los resultados de algunos de esos estudios.

Factores de riesgo, como el sexo, la edad o la artrosis, que han sido asociados a la formación de $\mathrm{OH}$ en cadera tras fracturas o artroplastias ${ }^{12}$, no pueden ser relacionados con la formación de $\mathrm{OH}$ en codo.

Se ha descrito en la literatura que el comienzo de la $\mathrm{OH}$ postraumática en el codo empieza a las 2 semanas aproximadamente después del traumatismo. Abrams et al. ${ }^{11}$ demuestran que en el $86 \%$ de los casos de su serie (47 de 55 pacientes) existen signos incipientes de $\mathrm{OH}$ en controles radiológicos realizados a las 2 semanas postraumatismo, estando presentes, en ese momento cronológico, dichos signos en el $100 \%$ de los casos que requirieron de cirugía escisional posterior (8 pacientes).

Hastings y Graham desarrollaron una clasificación para la $\mathrm{OH}$ en el codo $^{13}$ basada en la presencia radiológica de calcificaciones y fundamentalmente en la limitación en el grado de movilidad de dicha articulación secundaria a la 
Tabla 1 Factores de riesgo de $\mathrm{OH}$

\begin{tabular}{|c|c|c|}
\hline Autores/año & N. ${ }^{\circ}$ pacientes & Factores de riesgo/parámetros \\
\hline Guo et al., $2015^{7}$ & 457 & Inmovilización codo mayor 3 semanas \\
\hline Salazar et al., $2014^{8}$ & 46 & $\begin{array}{l}\text { Hipertensión arterial } \\
\text { Obesidad }\end{array}$ \\
\hline Wigger et al., $2014^{9}$ & 96 & $\begin{array}{l}\text { Luxación codo } \\
\text { Complejidad de fractura } \\
\text { Tiempo desde el traumatismo hasta la cirugía } \\
\text { Número de cirugías en las primeras } 4 \text { semanas }\end{array}$ \\
\hline Koh et al., $2013^{10}$ & 77 & Tiempo desde el trauma hasta la escisión de $\mathrm{OH}$ \\
\hline Lee et al., $2013^{1}$ (review) & 384 & $\begin{array}{l}\text { Baja movilidad articular previa escisión } \mathrm{OH} \\
\text { No inicio de RHB precoz pasiva tras escisión } \mathrm{OH} \\
\text { Retraso mayor a un año desde establecimiento } \mathrm{OH} \text { a su escisión }\end{array}$ \\
\hline Abrams et al., $2012^{11}$ & 55 & Fracturas de húmero distal \\
\hline
\end{tabular}

Se muestran los estudios y los factores de riesgo y parámetros que resultaron estar relacionados con la aparición de $\mathrm{OH}$, así como de su pronóstico en cuanto a resultados clínicos.

calcificación, asignando a la misma 3 grados, como se muestra en la tabla 2.

Se han intentado establecer métodos profilácticos para evitar la aparición de la $\mathrm{OH}$, con resultados limitados, mediante AINES (indometacina $75 \mathrm{mg} /$ día/6 semanas) y radioterapia preoperatoria, o bien en las primeras $48 \mathrm{~h}$ tras la cirugía ${ }^{14,15}$.

\section{Material y métodos}

Presentamos un análisis retrospectivo de los casos de $\mathrm{OH}$ en codo tratados quirúrgicamente en nuestro centro entre los años 2005 y 2015 . Se recogieron variables demográficas, antecedentes personales, evento traumático inicial, clínica, tiempo desde el traumatismo hasta el diagnóstico de $\mathrm{la} \mathrm{OH}$, tiempo desde el diagnóstico de $\mathrm{OH}$ hasta la cirugía escisional, seguimiento, recurrencia, y grados de movilidad en precirugía escisional y al final del seguimiento.

Con ello pretendemos mostrar nuestra experiencia en el tratamiento quirúrgico de esta patología.

\section{Resultados}

Obtuvimos 4 casos con un tiempo medio de seguimiento de 40 meses. En 2 casos el evento traumático fue la luxación de codo, y en los otros 2, la rotura del tendón del bíceps en su inserción distal. Tres de ellos debutaron clínicamente con limitación en la movilidad articular, y uno con déficit motor a nivel radial. Todos los casos fueron pacientes varones con una edad media de 48 años. El tiempo medio transcurrido desde el evento traumático hasta el diagnóstico de $\mathrm{OH}$ fue de 4 meses, realizándose el tratamiento quirúrgico escisional tras el diagnóstico igualmente a los 4 meses aproximadamente como media. Tres de los 4 casos recidivaron, apareciendo nuevas calcificaciones con repercusión clínica y funcional. En la tabla 3 se muestran los datos citados detallados en cada caso (fig. 1).

Los datos de repercusión clínica y resultados clínicos finales al final del seguimiento se muestran en la tabla 4. En los casos de afectación de movilidad articular, 2 de ellos tuvieron limitación en el rango articular de flexo extensión (tipo IIA de la clasificación de Hastings) y otro en pronosupinación (tipo IIB de la clasificación de Hastings). El caso que resta debutó con afectación neurológica con déficit de extensión del tercer y cuarto dedos de la mano.

Solo uno de los casos no recidivó, consiguiendo una mejora en balance articular de $95^{\circ}$ en flexo-extensión. Los otros 2 casos consiguieron ganancias articulares postoperatorias prácticamente completas, pero dada la recidiva de la calcificación volvieron a situaciones similares con ganancias de rango articular mínimas. En el caso con afectación neurológica (rotura y reinserción de tendón distal de bíceps), se practicó la exéresis de la $\mathrm{OH}$ y la neurólisis de la rama del nervio interóseo posterior afecta, consiguiendo mejorías clínicas progresivas que se truncaron tras la recidiva de la calcificación, volviendo a la situación inicial. Ello requirió de una nueva cirugía con exéresis de la calcificación, desanclaje del tendón distal de bíceps y tenodesis del mismo al músculo braquial. Actualmente se halla en

Tabla 2 Clasificación Hastings y Graham para la $\mathrm{OH}$ en codo

\begin{tabular}{|c|c|c|c|c|c|c|}
\hline \multicolumn{7}{|c|}{ Clasificación Hastings y Graham } \\
\hline \multirow{5}{*}{$\begin{array}{l}\text { Grado I } \\
\text { No causa } \\
\text { limitación } \\
\text { funcional }\end{array}$} & \multicolumn{3}{|c|}{ Grado II } & \multicolumn{3}{|c|}{ Grado III } \\
\hline & IIA & IIB & IIB & IIIA & IIIB & IIIC \\
\hline & Limitación & Limitación & Limitación & Anquilosis & Anquilosis & Anquilosis \\
\hline & Ext-flex & Prono-sup & en ambos & ext-flex & prono-sup & ambos \\
\hline & $>-30^{\circ} \mathrm{a}$ & $<50^{\circ} \mathrm{a}<50^{\circ}$ & planos & & & planos \\
\hline & $<130^{\circ}$ & & & & & \\
\hline
\end{tabular}


Tabla 3 Datos detallados de cada caso

\begin{tabular}{|c|c|c|c|c|c|c|c|c|c|}
\hline Caso & Sexo & Edad & AP & $\begin{array}{l}\text { Evento } \\
\text { traumático }\end{array}$ & Debut & $\begin{array}{l}\text { Tiempo desde } \\
\text { trauma a } \\
\text { diagnóstico de } \\
\text { OH }\end{array}$ & $\begin{array}{l}\text { Tiempo desde } \\
\text { diagnóstico } \mathrm{OH} \\
\text { hasta cirugía }\end{array}$ & Seguimiento & Recurrencia \\
\hline 1 & V & 47 & Ninguno & $\begin{array}{l}\text { Luxación } \\
\text { posterior } \\
\text { codo }\end{array}$ & $\begin{array}{l}\text { Rigidez } \\
\text { articular }\end{array}$ & 50 días & 5 meses & 15 meses & No \\
\hline 2 & V & 56 & HTA & $\begin{array}{l}\text { Rotura tendón } \\
\text { distal bíceps }\end{array}$ & $\begin{array}{l}\text { Déficit } \\
\text { motor RIP }\end{array}$ & 7 meses & 4 meses & 30 meses & Sí \\
\hline 3 & V & 55 & $\begin{array}{l}\text { HTA, } \\
\text { DM II }\end{array}$ & $\begin{array}{l}\text { Luxación } \\
\text { posterior } \\
\text { codo }\end{array}$ & $\begin{array}{l}\text { Rigidez } \\
\text { articular }\end{array}$ & 2 meses & 7 meses & 17 meses & Sí \\
\hline 4 & V & 36 & Ninguno & $\begin{array}{l}\text { Rotura tendón } \\
\text { distal bíceps }\end{array}$ & $\begin{array}{l}\text { Rigidez } \\
\text { articular }\end{array}$ & 6 meses & 1 mes & 8 años & Sí \\
\hline
\end{tabular}

AP: antecedente previo; DMII: diabetes mellitus tipo 2; HTA: hipertensión arterial; OH: osificación heterotópica; RIP: rama nervio interóseo posterior; V: varón.

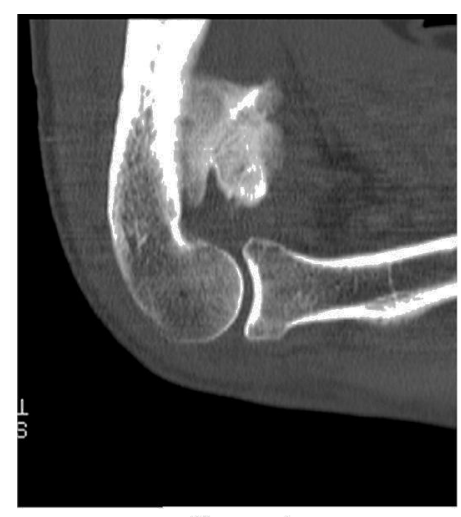

Caso 1

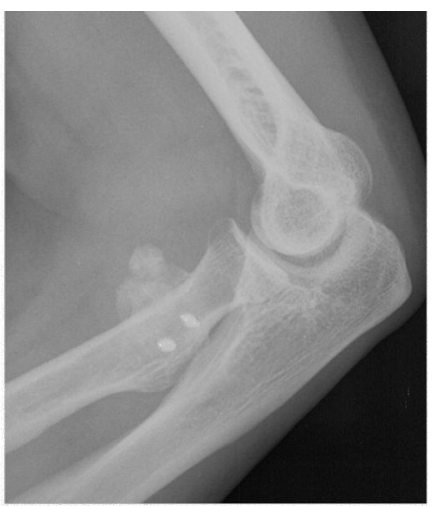

Caso 2

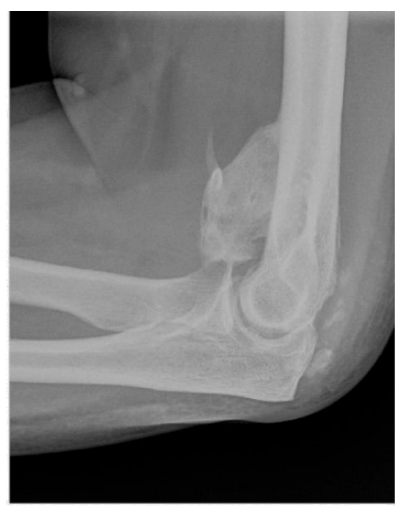

Caso 3

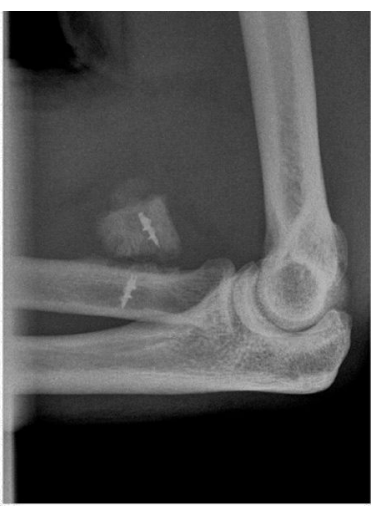

Caso 4

Figura 1 Imágenes radiológicas iniciales de la $\mathrm{OH}$ de cada uno de los casos.

Tabla 4 Clasificación, repercusión clínica y resultados finales

\begin{tabular}{llcccccc}
\hline Caso & Clasf. Hastings & Flex-ext. pre-IQ & Flex-ext. final & Ganancia & Pronosup. pre-IQ & Pronosup. final & Ganancia \\
\hline 1 & IIA & $105^{\circ} /-70^{\circ}$ & $130^{\circ} / 0^{\circ}$ & $25^{\circ} / 70^{\circ}$ & $75^{\circ} / 75^{\circ}$ & $75^{\circ} / 75^{\circ}$ & $0^{\circ} / 0^{\circ}$ \\
2 & - & $140^{\circ} / 0^{\circ}$ & $140^{\circ} / 0^{\circ}$ & $0^{\circ} / 0^{\circ}$ & $80^{\circ} / 80^{\circ}$ & $80^{\circ} / 80^{\circ}$ & $0^{\circ} / 0^{\circ}$ \\
3 & IIA & $90^{\circ} /-30^{\circ}$ & $84^{\circ} /-39^{\circ}$ & $-6^{\circ} /-9^{\circ}$ & $60^{\circ} / 80^{\circ}$ & $68^{\circ} / 80^{\circ}$ & $8^{\circ} / 0^{\circ}$ \\
4 & IIB & $120^{\circ} / 0^{\circ}$ & $120^{\circ} / 0^{\circ}$ & $0^{\circ} / 0^{\circ}$ & $20^{\circ} / 80^{\circ}$ & $28^{\circ} / 80^{\circ}$ & $8^{\circ} / 0^{\circ}$ \\
\hline
\end{tabular}

fase de recuperación. Este caso recibió terapia profiláctica tras la primera cirugía de exéresis mediante indometacina $75 \mathrm{mg} /$ día/ 6 semanas.

\section{Discusión}

Llama la atención el alto índice de recidiva en nuestra serie, con una tasa del $75 \%$ frente al $10-24 \%$ descrito en la literatura ${ }^{10}$, lo cual implica que nuestros resultados finales se vean afectados seriamente. Sin embargo, en el caso de no recidiva la ganancia articular en flexo extensión de $95^{\circ}$ está por encima de las ganancias medias descritas en la literatura ${ }^{1}$. Tradicionalmente muchos autores recomendaban esperar 12-18 meses desde el diagnóstico de la $\mathrm{OH}$ hasta el momento de su escisión. Sin embargo, actualmente se ha descrito que retrasos mayores a un año repercuten negativamente en los resultados finales ${ }^{1}$. Igualmente, Koh et al. ${ }^{10}$ han establecido el punto de corte de 19 meses desde el traumatismo inicial hasta la exéresis de la $\mathrm{OH}$ como cifra a partir de la cual los resultados finales empeoran. Curiosamente, en nuestra serie todos los casos han cumplido estas 2 últimas recomendaciones, siendo los tiempos medios transcurridos desde el evento traumático hasta la exéresis de $\mathrm{OH}$ de 8 meses, realizándose el tratamiento quirúrgico escisional tras el diagnóstico de la misma como media aproximadamente a los 4 meses.

En cuanto al tratamiento profiláctico, uno de los casos (caso 2 ) recibió profilaxis con indometacina tras la exéresis 
de la $\mathrm{OH}$, recidivando y teniendo que ser reintervenido a los 9 meses.

El 50\% de nuestros casos presentaban HTA, antecedente determinado según algunos autores como factor de riesgo ${ }^{8}$.

Como traumatismo inicial, 2 casos fueron luxaciones de codo, situación frecuentemente descrita en la literatura, y ambos fueron tratados con inmovilización mediante fijador externo durante 6 semanas. Recientemente, se han cifrado las 3 semanas de inmovilización como factor de riesgo para desarrollar $\mathrm{OH}^{7}$, el cual no se ha cumplido en nuestros 2 casos.

En los 2 casos restantes, la $\mathrm{OH}$ apareció posteriormente a la reinserción de tendón distal del bíceps tras su rotura. Existe un caso descrito de $\mathrm{OH}$ tras reinserción de tendón distal de bíceps mediante técnica endobutton (Smith and Nephew Endoscopy, Andover, MA) ${ }^{16}$ sin presentar recidiva. Nuestros casos fueron tratados mediante anclaje con arpón óseo a nivel de la tuberosidad bicipital del radio, recidivando ambos.

La limitación de nuestro estudio es su naturaleza retrospectiva, así como el tamaño reducido de la muestra al ceñirnos únicamente a los casos de $\mathrm{OH}$ tratados quirúrgicamente. Con él no pretendemos establecer por tanto cifras ni conclusiones, pero sí queremos mostrar y contrastar nuestros datos y resultados con los de la literatura más actual.

\section{Conclusión}

Si bien no podemos establecer conclusiones sólidas dada la naturaleza de nuestro estudio y su tamaño muestral, nuestros resultados convergen con los de la literatura en cuanto a factores de riesgo y resultados funcionales finales tras la cirugía escisional. Sin embargo, llama la atención el alto índice de recidiva en nuestra serie, con una tasa del $75 \%$ frente al $10-24 \%$ descrito en la literatura, por lo que creemos que es necesario seguir profundizando en la investigación clínica sobre la etiología y causas de recidiva de este fenómeno.

\section{Conflicto de intereses}

Los autores declaran no tener ningún conflicto de intereses.

\section{Bibliografía}

1. Lee E, Namdari S, Hosalkar HS, Keenan MA, Baldwin KD. Clinical results of the excision of heterotopic bone around the elbow: A systematic review. J Shoulder Elbow Surg. 2013;22: 716-22.

2. Abrams GD, Bellino MJ, Cheung EV. Risks factors for development of heterotopic ossification of the elbow affecter fracture fixation. J Shoulder Elbow Surg. 2012;21:1550-4.

3. Ilahi OA, Strausser DW, Gabel GT. Post-traumatic heterotopic ossification about elbow. Orthopedics. 1998;21: 265-8.

4. Bauer AS, Lawson BK, Bliss RL, Dyer GS. Risk factors for postraumatic heterotopic ossification of the elbow: Case-control study. J Hand Surg Am. 2012;37:1422-9.

5. Foruria AM, Augustin S, Morrey BF, Sánchez-Sotelo J. Heterotopic ossification after surgery for fractures and fractures dislocations involving the proximal aspect of the radius or ulna. J Bone Joint Surg Am. 2013;95:e66.

6. Morrey B, Harter GD. Ectopic ossification about the elbow. En: Morrey B, Sanchez-Sotelo J, editores. The elbow and its disorders. 4. ${ }^{\mathrm{a}}$ ed. Philadelphia: Saunders; 2009. p. 472-84.

7. Guo Q, He D, Sun N, Zha Y, Jiang X. Retrospective analysis of 553 patients with posttraumatic elbow stiffness. Zhonghua Wai Ke Za Zhi. 2015;53:85-9.

8. Salazar D, Golz A, Israel H, Marra G. Heterotopic ossification of the elbow treated with surgical resection: Risk factors, bony ankylosis, and complications. Clin Orthop Relat Res. 2014;472:2269-75.

9. Wiggers JK, Helmerhorst GT, Brouwer KM, Niekel MC, Nunez F, Ring D. Injury complexity factors predict heterotopic ossification restricting motion after elbow trauma. Clin Orthop Relat Res. 2014;472:2162-7.

10. Koh KH, Lim TK, Lee HI, Park MJ. Surgical treatment of elbow stiffness caused by post-traumatic heterotopic ossification. J Shoulder Elbow Surg. 2013;22:1128-34.

11. Abrams GD, Bellino MJ, Cheung EV. Risk factors for development of heterotopic ossification of the elbow after fracture fixation. J Shoulder Elbow Surg. 2012;21:1550-4.

12. Ahrengart L, Lindgren U. Functional significance of heterotopic bone formation after total hip arthroplasty. J Arthroplasty. 1989;4:125-31.

13. Hastings $H$, Graham TJ. The classification and treatment of heterotopic ossification about the elbow and forearm. Hand Clin. 1994;10:417-37.

14. Baird EO, Kang QK. Prophylaxis of heterotopic ossification - an updated review. J Orthop Surg Res. 2009;20:12.

15. Jiang J, Fang T, Chen L, Chen Y, Sun B. Efficacy and prognostic factors of preoperative radiation therapy of elbow arthrolysis. Zhonghua Yi Xue Za Zhi. 2014;94:1003-5.

16. Agrawal V, Stinson MJ. Case report: Heterotopic ossification after repair of distal tendon rupture utilizing a single incision Endobutton technique. J Shoulder Elbow Surg. 2005;14: 107-9. 